\title{
High Performance Liquid Chromatography of Fluorescein Isothiocyanate Isomers and Intermediate Products
}

\author{
Koji Muramoto*, Hisao KamiYa*, Hiroshi Kawauchi* \\ and Hiroshi Meguro** \\ *School of Fisheries Sciences, Kitasato University, Sanriku, Iwate 022-01 \\ **Faculty of Agriculture, Tohoku University, Tsutsumidori-amamiya, Sendai 980
}

\begin{abstract}
Fluorescein isothiocyanate (FITC) isomers and the intermediate products have been separated by reversed phase high performance liquid chromatography (HPLC), using a linear gradient formed from acetone and 0.01 $\mathrm{M}$ sodium phosphate buffer $(\mathrm{pH} 7.0)$ or an isocratic elution of $40 \%$ acetone in $0.1 \mathrm{M}$ pyridine-acetic acid $(\mathrm{pH}$ 7.0). This HPLC system was used to evaluate commercial FITC products. A method for the purification of the FITC product by HPLC was also described.
\end{abstract}

Keywords Fluorescein isothiocyanate, high performance liquid chromatography, fluorescent reagent

Fluorescein isothiocyanate(FITC) is the most commonly used fluorescent reagent in immunofluorescenct techniques ${ }^{1}$ and chemical modification of proteins. ${ }^{2}$ We have applied it to the amino acid sequence analysis of peptides and proteins. ${ }^{3}$ FITC is available from a number of commercial sources and used without attention to its purity. However, the commercially available FITC products have been shown great variation in purity.$^{4-7}$ The low purity of these products remains a problem in their optimal use in protein labeling, because the purity is a major factor in determining the conditions necessary to obtain the optimum degree of labeling, and in preventing nonspecific labeling. ${ }^{6-8}$ Contamination of the isomers results in misestimation of fluorescein/protein ratio of the labeled protein, since the spectroscopic characteristics of them are different. ${ }^{9}$ Particularly, highly purified FITC must be used for the purpose of the identification of labeled products by chromatography.

Considerable effort has been made to obtain pure FITC and to evaluate the chemical purity of FITC products. FITC is prepared by treatment of aminofluorescein(AF) with thiophosgene. ${ }^{10}$ The amines are obtained by reduction of the corresponding nitrofluoresceins(NF), which are the main products from the condensation reaction of 4-nitrophthalic acid with resorcinol. ${ }^{11,12}$ This reaction yields two NF isomers. They are separated by means of the differential solubility of their diacetates. Therefore contamination of two isomers in FITC preparation is due to incompleteness of the separation of the nitrofluorescein diacetates(NFDA). By-products in the reduction of NF can be removed by ion-exchange chromatography ${ }^{13}$, but not by recrystallization. Hydro- gen chloride formed during reaction of AF with thiophosgene results in the formation of an unstable hydrochloride of FITC, that causes the discrepancy of the elemental analysis of the hydrochloride. Hydrogen chloride-free FITC is obtainable by addition of calcium carbonate to the reaction mixture. ${ }^{14}$

Since there is a wide variation in the purities of commercial product, many methods have been reported for evaluating FITC content. McKinney et al. ${ }^{4}$ used the infrared band near $2000 \mathrm{~cm}^{-1}$ to estimate FITC content. Wilderspin and Green ${ }^{7}$ showed the rapid spectrophotometric titration of the isothiocyanate with mercaptoethanol. Several groups investigated the purity based upon the bovine serum albumin or immunoglobulin labeling efficiency.9,15,16 However, none of these methods gives information of the presence of the isomers. On the other hand, FITC isomers and the intermediate products can be separated by paper chromatographys, silica gel thin layer chromatography ${ }^{6,8}$ and silicic acid column chromatography $^{8}$, in spite of the troublesome of the quantitative analysis.

In this paper, we describe the use of reversed phase high performance liquid chromatography(HPLC) for the qualitative and quantitative evaluation of FITC isomers and the intermediate products together with a simplified method for the purification of a commercial FITC product.

\section{Experimental}

Synthesis of FITC

FITC isomers and the intermediate products were 


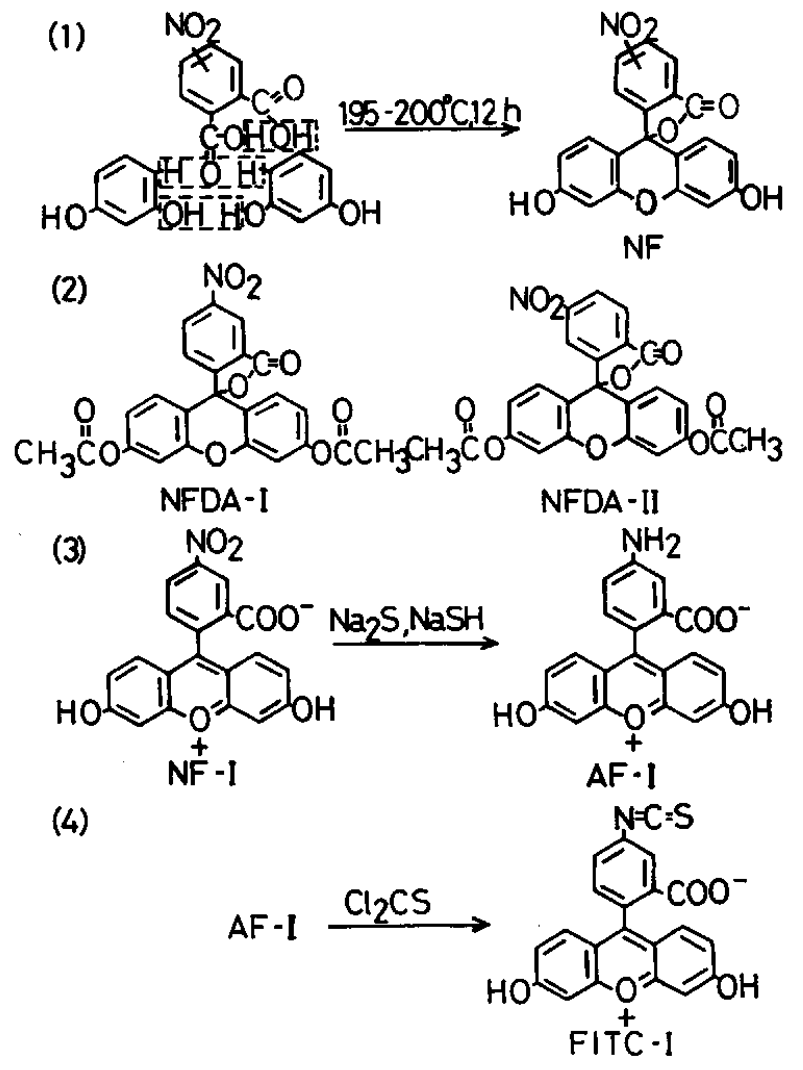

Fig. 1 The scheme of the synthesis of fluorescein isothiocyanate. (1) Condensation of 4-nitrophthalic acid and resorcinol to NF. Ref. 11. (2) Separation of NF-I and NF-II as diacetates. (3) Reduction of NF to the corresponding AF. Ref. 12. (4) Conversion of AF to the corresponding FITC. Ref. 10 and 14. NF, nitrofluorescein; NFDA, nitrofluorescein diacetate; AF, aminof luorescein; FITC: fluorescein isothiocyanate; I, isomer I; II, isomer II.

synthesized as outlined in Fig. 1. AF isomers were purified by DEAE cellulose chromatography according to the method of Dandliker and Alonso..$^{13}$ Conversion of the AF to FITC was carried out with freshly distilled thiophosgene in the presence of calcium carbonate as described by Sinsheimer et al. ${ }^{14}$ Recrystallization from acetone-petroleum ether gave an orange yellow crystal of FITC-I which decomposed above $193^{\circ} \mathrm{C}$. Anal. Calcd. for $\mathrm{C}_{21} \mathrm{H}_{11} \mathrm{NO}_{5} \mathrm{~S}$ (389.4): C, 64.78; $\mathrm{H}, 2.85 ; \mathrm{N}$, 3.60; S, 8.23. Found: C, 64.54; H, 2.69; N, 3.44; S, 8.04. FITC-II decomposed above $198^{\circ} \mathrm{C}$. Anal. Found: C, 64.54; H, 2.98; N, 3.32; S, 8.08. The elementary analyses of all the intermediate products agreed with the theoretical values within $0.3 \%$.

\section{HPLC}

Chromatography equipment consisted of a Jasco Tri Rotor SR pump, a Jasco Tri Rotor SR2 controller, a Jasco Uvidec-100-IV variable wavelength detector (490 $\mathrm{nm})$, a Jasco FP-550A spectrofluorometer $\left(\lambda_{\mathrm{ex}}=490\right.$ $\mathrm{nm}, \lambda_{\mathrm{em}}=515 \mathrm{~nm}$ ), and a SIC Chromatocorder 11 .
A saturator column $(4.6 \times 50 \mathrm{~mm})$ dry-packed with Wakogel LC ODS-30K $(30 \mu \mathrm{m}$ particles, Wako Junyaku) and an ODS Hypersil (3 $\mu \mathrm{m}$ particles, Shadon Southern Products) column (4.6×50 mm) slurry packed in our laboratory were connected through a valve injector, and were housed within an oven compartment maintained at $40^{\circ} \mathrm{C}$. The solvent systems were (A) $10 \mathrm{mM}$ sodium phosphate buffer $(\mathrm{pH} 7.0)$ and (B) $10 \mathrm{mM}$ sodium phosphate buffer $(\mathrm{pH} 7.0)$-acetone $(80: 20, \mathrm{v} / \mathrm{v})$. The flow rate was $1.0 \mathrm{ml} / \mathrm{min}$. The gradient was linear over $30 \mathrm{~min}$ from 10 to $100 \%$ solvent B. Samples (20 ng each) dissoived in acetone were injected.

\section{Purification of FITC}

FITC and intermediate products (50 $\mathrm{ng}$ each) were separated on a Jasco Fine Sil ODS-5 column $(5 \mu \mathrm{m}$ particles, $4.6 \times 250 \mathrm{~mm}$ ) using an isocratic elution. The solvent was $40 \%$ acetone in $0.1 \mathrm{M}$ pyridine-acetic acid (pH 7.0). The column temperature was maintained at $30^{\circ} \mathrm{C}$. The flow rate was $1.0 \mathrm{ml} / \mathrm{min}$. This HPLC system was applied to the purification of a commercial product. FITC-A ( $25 \mathrm{mg}$ ) dissolved in $2 \mathrm{ml}$ of acetone was passed through a Sep-Pak C18 filter (Waters Assoc.) and diluted with $4 \mathrm{ml}$ of the running buffer. One milliliter of the solution was injected to the column for a run. The elution peaks were detected at $490 \mathrm{~nm}$ and lyophilized to give $7.3 \mathrm{mg}$ of FITC-I and $4.5 \mathrm{mg}$ of FITC-II.

\section{Evaluation of purified FITC}

To evaluate the purified FITC, the amino-terminal sequence of egg white lysozyme was analyzed by the FITC method. ${ }^{3}$ The protein $(5 \mathrm{nmol})$ was dissolved in $100 \mu \mathrm{l}$ of $60 \%$ aqueous pyridine containing $200 \mathrm{nmol}$ of either the synthesized FITC-I or the purified commercial FITC-I. Yielded FITC derivatives of amino acids were dissolved in methanol and subjected to HPLC analysis. ${ }^{17}$

\section{Results and Discussion}

The synthesized FITC isomers and the intermediate products gave single peaks and were separated from each other by reversed phase HPLC as shown in Fig. 2 . We used this HPLC system to evaluate two commercial FITC products. HPLC revealed that FITC-A was mixtures of FITC isomers. FITC-B contained small amounts of isomer II, as indicated by the chromatogram. FITC contents of FITC-B and FITC-A were $81.3 \pm 6.1 \%$ and $77.4 \pm 4.5 \%$ (FITC-I, 36.7 $\pm 2.9 \%$; FITCII, 40.7 $\pm 1.7 \%$ ) (mean \pm S.E., $n=3$ ), respectively. Under the conditions developed in this investigation, HPLC can be used, with practice, to evaluate FITC samples quantitatively. The separation and identification of isomers is possible with slight effort. Moreover, this method is suitable for supervising each step in the synthesis of FITC and provides a means of determining 


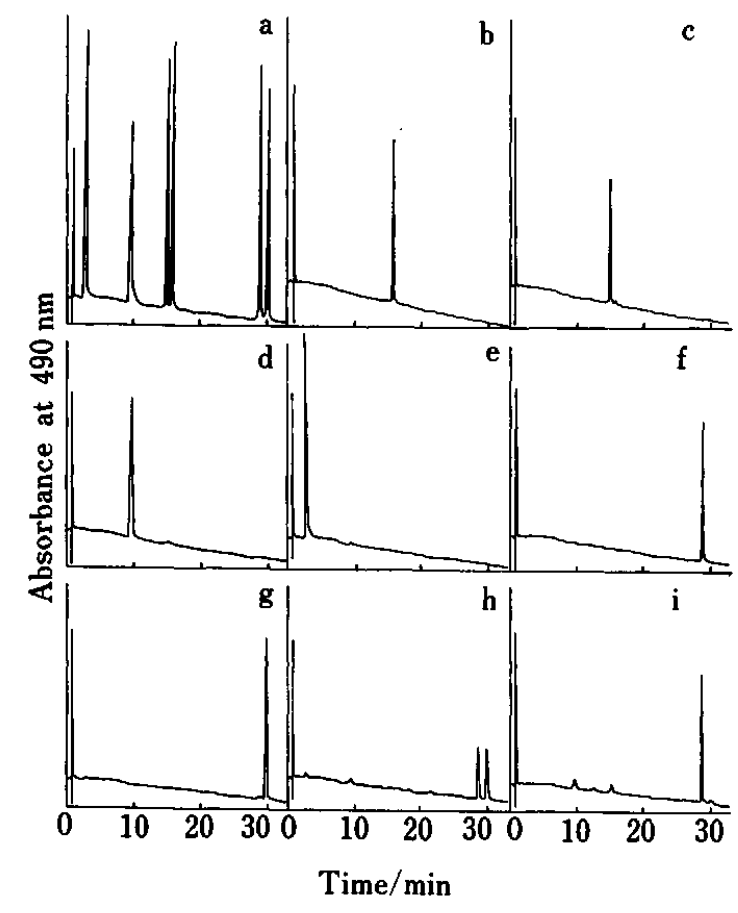

Fig. 2 Chromatograms of fluorescein isothiocyanates and intermediate products on an ODS Hypersil column $(3 \mu \mathrm{m}$ particles, $4.6 \times 50 \mathrm{~mm})$. Operating conditions: column temperature $40^{\circ} \mathrm{C}$; gradient elution, mobile phase: $A=10 \mathrm{mM}$ sodium phosphate buffer, pH $7.0 ; B=10 \mathrm{mM}$ sodium phosphate buffer, pH 7.0 -acetone $(80: 20, \mathrm{v} / \mathrm{v})$; linear gradient from 10 to $100 \%$ solvent $B$ in $30 \mathrm{~min}$ at a flow rate of 1.0 $\mathrm{ml} / \mathrm{min}$. Injected amount: $20 \mathrm{ng}$ each. a, standard mixture; b, NF-I; c, NF-II; d, AF-I; e, AF-II; f, FITC-I; g, FITC-II; h, commercial FITC-A; i, commercial FITC-B.

accurately the shelf life of FITC products under various storage conditions.

The above result encouraged us to purify commercial FITC by reversed phase HPLC, because a simple method for the purification of commercial FITC was expected to release investigator from the laborious task to synthesize pure FITC. We adopted an isocratic elution using a volatile buffer in the preparative purification for its easiness to operate and remove. FITC isomers and their intermediate products were separated sufficiently under this condition as shown in Fig. 3. Figure 4 shows the preparative separation of commercial FITC-A. The yields of FITC-I and FITC-II were 29.2 and $18 \%$, respectively. Each of the purified FITC isomers showed a single peak by rechromatography. The content was estimated to be $74 \pm 7.3 \%$ for FITC-I and $82.7 \pm 2.7 \%$ for FITC-II. To evaluate the quality of the purified FITC, we analyzed the amino-terminal sequence of lysozyme using both synthesized and purified FITC-I. In the case that the purified FITC contains either isomer or oxidized form, there should be differences in the chromatograms resulting from both reagents; that is, complex elution peaks or low yields of fluorescein thiohydantoin(FTH) amino acids

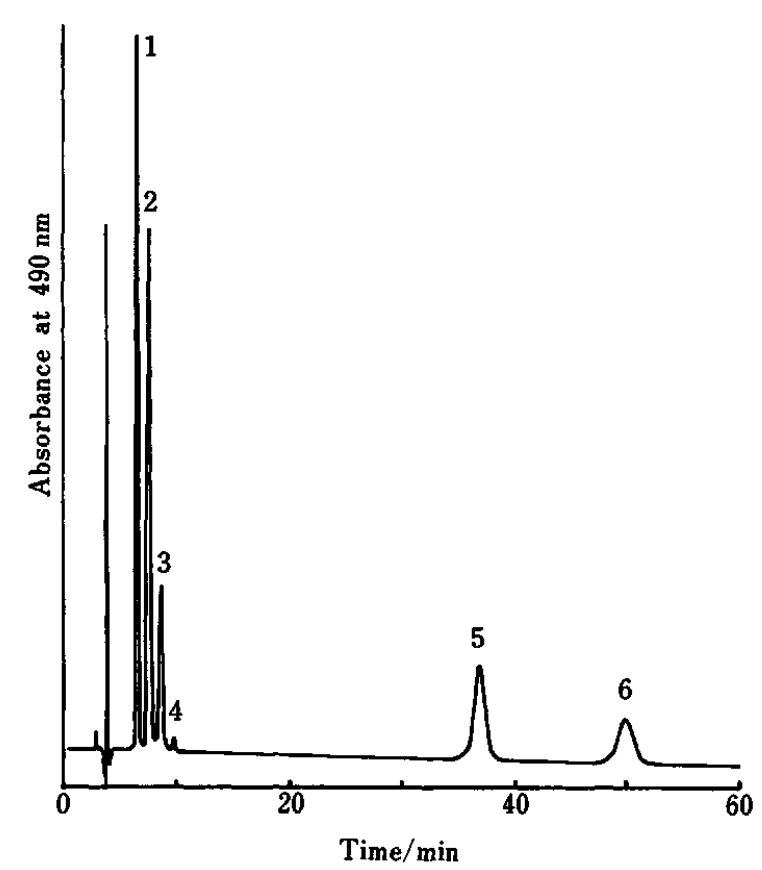

Fig. 3 Separation of fluorescein isothiocyanates and intermediate products on a Jasco Fine Sil ODS-5 column ( $5 \mu \mathrm{m}$ particles, $4.6 \times 250 \mathrm{~mm}$ ). Operating conditions: column temperature $30^{\circ} \mathrm{C}$; isocratic elution, mobile phase: $0.1 \mathrm{M}$ pyridine-acetic acid, pH 7.0acetone $(60: 40, \mathrm{v} / \mathrm{v})$; flow rate: $1.0 \mathrm{ml} / \mathrm{min}$; injected amount: $50 \mathrm{ng}$ each. Peak identification: $1=\mathrm{NF}-\mathrm{II}$; $2=$ NF-I; $3=$ AF-I; $4=$ AF-II; $5=$ FITC-I; $6=$ FITC-II.

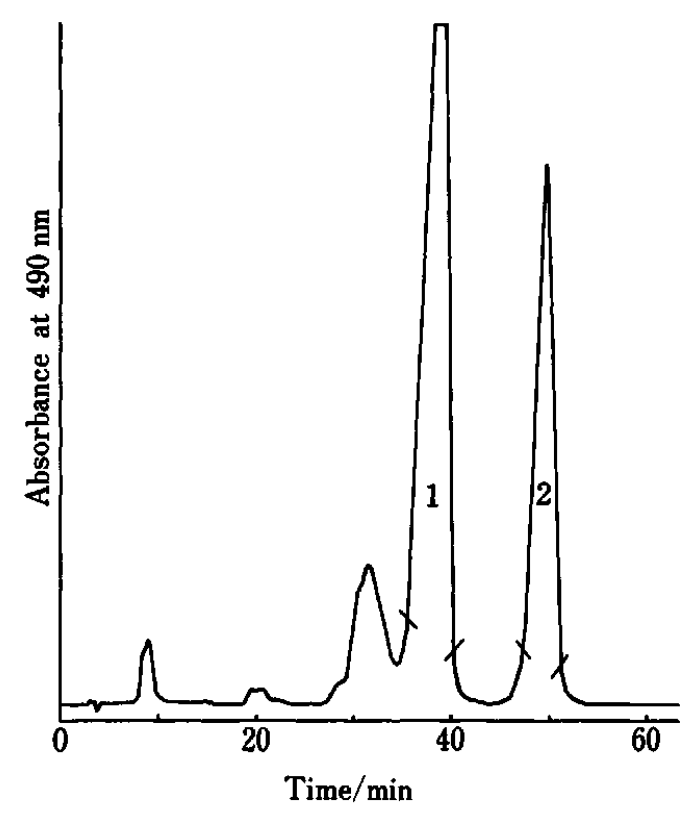

Fig. 4 Purification of fluorescein isothiocyanate by reversed phase HPLC. Conditions are the same as in Fig. 3. Approximately $1 \mathrm{mg}$ of commercial FITC-A was applied. Peak identification: $1=$ FITC-I; $2=$ FITC-II. 


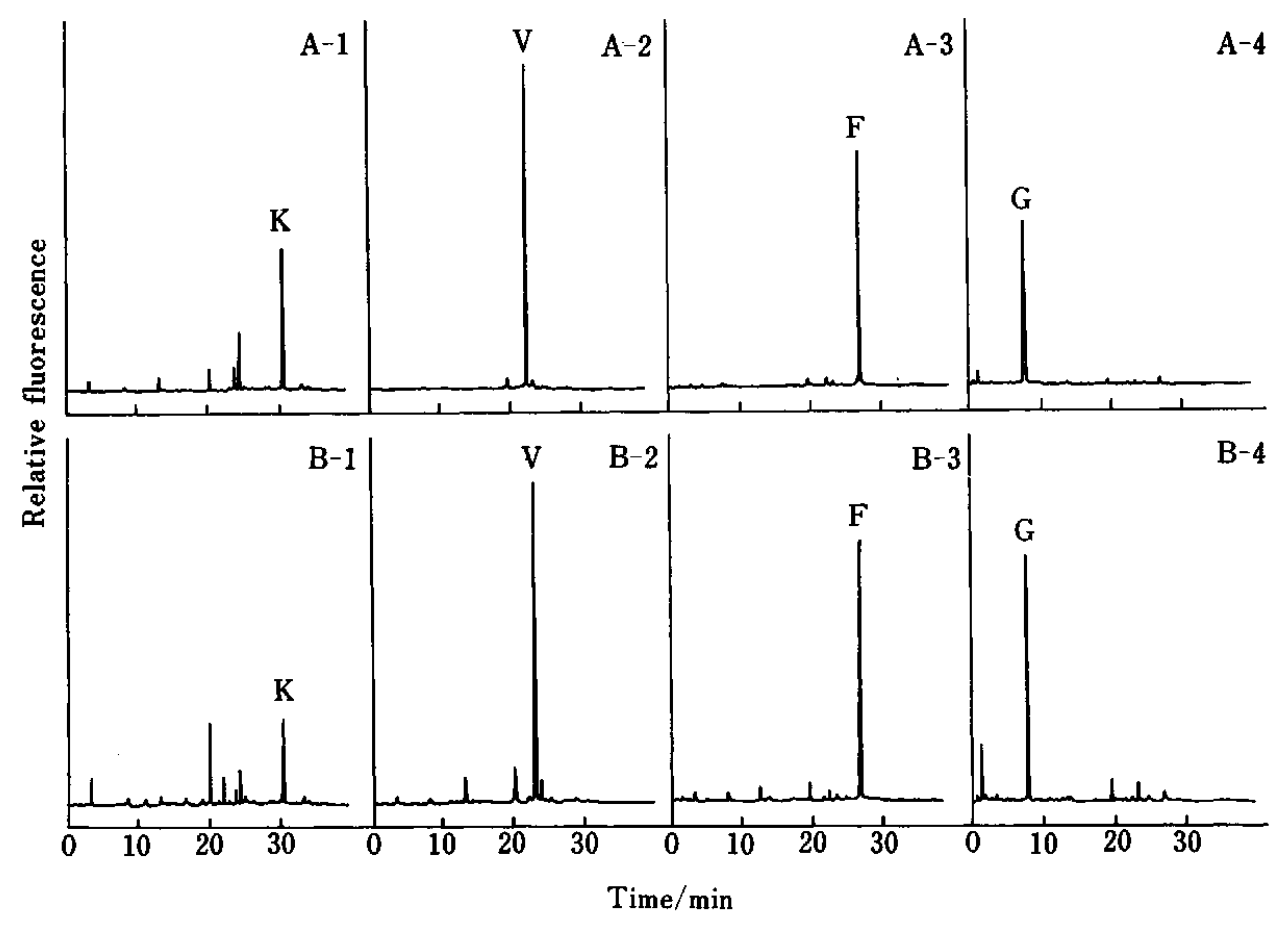

Fig. 5 HPLC chromatograms from the sequencing of $5 \mathrm{nmol}$ of egg white lysozyme (Lys-ValPhe-Gly $\cdots)$. Chromatographic conditions are the same as in Fig. 2 except that the column temperature was $60^{\circ} \mathrm{C}$ and the linear gradient was from 15 to $70 \%$ solvent $\mathrm{B}$. About $0.1 \%$ of the FTH derivatives derived from the sequencing was subjected to HPLC analysis. Figures in the panels are the cycle number of sequencing with synthesized FITC (A) and purified FITC (B). Peak identification: $K=$ FTH-Lys; V =FTH-Val; F =FTH-Phe; G=FTH-Gly.

should be observed. The HPLC chromatograms of FTH-amino acids obtained from the sequencing are given in Fig. 5. No significant difference was observed between the two FITC-I. This result indicates that the use of this HPLC system should be of considerable value to workers attempting to make purer preparations of labeled proteins. FITC can be readily purified on a larger scale using a preparative column. This system is also applicable to the purification of $\mathrm{AF}$ which is a stable intermediate product and can be converted to FITC according to need.

\section{References}

1. A. Kawamura, Jr. and Y. Aoyama, "Immunofluorescence in Medical Science", University of Tokyo Press (1982).

2. K. Muramoto and H. Kawauchi, Tanpakushitsu Kakusan Koso, 29, 735 (1984).

3. K. Muramoto, H. Kawauchi and K. Tuzimura, Agric. Biol. Chem., 42, 1559 (1978).

4. R. M. McKinney, J. T. Spillane and G. W. Pearce, Anal. Biochem., 7, 74 (1964).

5. H. S. Corey, Jr. and R. M. McKinney, Anal. Biochem., 4, 57 (1962).
6. G. Steinbach, Acta histochem., 49, 19 (1974).

7. A. F. Wilderspin and N. M. Green, Anal. Biochem., 132, 449 (1983).

8. Y. Nagai and A. Kawamura, Jr., Japan. J. Exp. Med., 43, 53 (1973).

9. G. Steinbach and H. von Mayersbach, Acta histochem., 55, 110 (1976).

10. J. L. Riggs, R. J. Seiwald, J. H. Burckhalter, C. M. Downs and T. G. Metcalf, Amer. J. Pathol., 34, 1081 (1958).

11. A. H. Coons and M. H. Kaplan, J. Expt. Med., 91, 1 (1950).

12. R. M. McKinney, J. T. Spillane and G. W. Pearce, J. Org. Chem., 27, 3986 (1962).

13. W. B. Dandliker and R. Alonso, Immunochemistry, 4, 191, (1967).

14. J. E. Sinsheimer, V. Jagodic and J. H. Burckalter, Anal. Biochem., 57, 227 (1974).

15. T. Tokumaru, J. Immunol., 89, 195 (1962).

16. R. M. McKinney, J. T. Spillane and G. W. Pearce, J. Immunol., 93, 232 (1964).

17. K. Muramoto, H. Kamiya and H. Kawauchi, Anal. Biochem., 141, 446 (1984).

(Received June 24, 1985) (Accepted September 5, 1985) 